\title{
Postbrushing and Fine-Needle Aspiration Biopsy Follow-Up and Treatment Options for Patients With Pancreatobiliary Lesions: \\ The Papanicolaou Society of Cytopathology Guidelines
}

\author{
Daniel Kurtycz, M.D., ${ }^{1 *}$ Z. Laura Tabatabai, M.D., ${ }^{2}$ Claire Michaels, M.D., ${ }^{3}$ \\ Nancy Young, M.D., ${ }^{4}$ C. Max Schmidt, M.D., ${ }^{5}$ James Farrell, M.D., \\ Deepak Gopal, M.D., ${ }^{7}$ Diane Simeone, M.D., ${ }^{8}$ Nipun B. Merchant, M.D., ${ }^{9}$ \\ Andrew Field, M.B.B.S., ${ }^{10}$ and Martha Bishop Pitman, M.D. ${ }^{11}$
}

The papanicolaou society of cytopathology (PSC) has developed a set of guidelines for pancreatobiliary cytology including indications for endoscopic ultrasound (EUS) guided fine-needle aspiration (FNA) biopsy, techniques of EUS-FNA, terminology and nomenclature for pancreatobiliary cytology, ancillary testing, and postprocedure management. All documents are based on the expertise of the authors, a review of the literature, discussions of the draft document at several national and international meetings over an 18 month period and synthesis of online comments of the draft document on the PSC web site [www.papsociety.org]. This document selectively presents the results of these discussions and focuses on the follow-up and treatment options for patients after procedures performed for obtaining cytology samples for the evaluation of biliary strictures and solid and cystic masses in the pancreas. These recommendations follow the six-tiered terminology and nomenclature scheme proposed by Committee III. Diagn. Cytopathol. 2014;42:363-371. @ 2014 Wiley Periodicals, Inc.

Key Words: pancreas; cytology; management; treatment; PSC; guidelines
The successful performance of any medical procedure is operator dependent. In the case of bile duct brushings and/ or endoscopic ultrasound guided fine-needle aspiration (EUS-FNA) of pancreatic lesions, a successful outcome depends on the performance of a dedicated team. The interventionalist must accurately identify and biopsy the target tissue as well as obtain sufficient cellular material for interpretation. The cytologist must make a good quality, interpretable smear or other satisfactory preparation, be it a liquid based cytology, cell-block or sample for genetic analysis. Finally, the cytopathologist who analyzes the sample must be well-trained and experienced in interpretation of rapid on site evaluations (ROSE) and final slides, or at least have adequate training and the backup of senior more experienced colleagues. Additionally, the diagnosis of pancreatic lesions is helped by a multidisciplinary approach involving radiologic and clinical input. This
${ }^{1}$ Department of Pathology, University of Wisconsin, Wisconsin State Laboratory of Hygiene, Madison, Wisconsin

${ }^{2}$ Department of Pathology, UCSF, San Francisco, California

${ }^{3}$ Department of Pathology, Case Western Reserve University, Cleveland, Ohio

${ }^{4}$ Department of Pathology, Albert Einstein College of Medicine, Bronx, New York

${ }^{5}$ Department of Surgery, Indiana University, Indianapolis, Indiana

${ }^{6}$ Department of Medicine, UCLA School of Medicine, Los Angeles, California

${ }^{7}$ Division of Gastroenterology, Department of Medicine, University of Wisconsin, Madison, Wisconsin
${ }^{8}$ Department of Surgery, University of Michigan, Michigan

${ }^{9}$ Division of Surgical Oncology, Department of Medicine, Vanderbilt University, Vanderbilt, Nashville, Tennessee

${ }^{10}$ Department of Pathology, St. Vincent's Hospital, Sydney, Australia

${ }^{11}$ Department of Pathology, Massachusetts General Hospital, Harvard Medical School, Boston, Massachusetts

*Correspondence to: Dr. Daniel Kurtycz, MD, Department of Pathology, University of Wisconsin, Wisconsin State Laboratory of Hygiene, 465 Henry Mall, Office 429a, WI, USA. E-mail: dkurtycz@wisc.edu Received 15 January 2014; Accepted 15 January 2014

DOI: $10.1002 /$ dc. 23121

Published online in Wiley Online Library (wileyonlinelibrary.com). 
multidisciplinary component is often not recognized or investigated in the various studies published in the literature regarding the sensitivity, specificity, and predictive values of pancreatic/biliary duct brushings and EUSFNA. ${ }^{1-4}$

The clinician managing the patient needs to have confidence in the cytologic diagnosis. To this end, the cytopathology team should regularly demonstrate their findings at multidisciplinary case conferences and, where appropriate, during ROSE. A double-headed microscope can quickly acquaint the interventionalist with the yield of their brushings or FNA. The interventionalist can make this a two-way learning experience by sharing the radiographic images of the pancreatic lesion being biopsied. Imaging will inevitably give valuable clues to the existence, diagnosis and extent of the pancreatic lesion. This information will narrow the diagnostic possibilities and help optimize the cytologic interpretation. Dual phase, contrast enhanced spiral computed tomography (CT) can establish the solid, cystic, confined, or infiltrative nature of a lesion, as well as provide information about potential metastatic sites in nearby organs or regional lymph nodes. ${ }^{5}$ In cases where actual tissue sampling is necessary, core needle biopsy may need to be performed. ROSE of a cytologic touch preparation from the core biopsy may prove to be very valuable. The results can be communicated directly at the same procedure, just as in an FNA, ROSE of a touch preparation can confirm whether the interventionalist has obtained a significant sample, provide direction to obtain additional material if the original biopsy was not successful, or if material is needed for ancillary stains or procedures.

Cytologic sampling can be achieved by endoscopic retrograde duct brushing (DB), percutaneous fine-needle aspiration (pFNA), or EUS guided FNA (EUS-FNA). For cystic pancreatic lesions greater than $2 \mathrm{~cm}$, a cytobrush passed down a 19-gauge needle may be added to routine FNA. In certain instances, core biopsy to obtain adequate tissue can be performed with a 22-25 Gauge FNA needle, although it is more usual to gain core biopsy type samples with needles 19 gauge and larger. ${ }^{6-9}$

The purpose of this workgroup effort is to discuss the options regarding and management of patients following cytologic diagnoses made by biliary brushing or FNA cytology, using the terminology developed by the Pancreatic/Biliary Guideline Committee III.

\section{Follow-Up in Relation to the Proposed Diagnostic Terminology}

\section{Nondiagnostic}

A nondiagnostic cytology sample is defined as a sample that is inadequate for interpretation due to whatever cause; there is not enough cytological material to make any diagnostic comment. For pancreatic lesions, a simple cell count to determine sample adequacy as is done in thyroid FNA is not enough. Obtaining pancreatic cytology samples is much more involved and often has more serious implications than FNA of superficial sites. The cytologic diagnosis must explain and be consistent with the clinical and radiologic findings no matter the number of cells or cellular groups in the cytology sample. It is suggested that only up to 4 passes be attempted, after which the FNA procedure's opportunity to obtain diagnostic material decreases. This has demonstrated in the pancreas and in other sites. ${ }^{10-13}$

With an inadequate FNA or brushing, clinical management becomes solely dependent on the clinical and imaging findings and is more insecure. If diagnostic confidence in the imaging and clinical findings are high, the team may elect to proceed directly to laparotomy to obtain diagnostic material via tru-cut needle, incisional, or excisional biopsy.

If the first attempt at cytological diagnosis is by bile duct brushings, then a second bile brushing attempt or EUS-FNA of any mass lesion of the distal hepatic, mid/ proximal bile duct, or intrapancreatic common bile duct should be attempted. If the first attempt was by percutaneous FNA then it may be most reasonable to use EUSFNA, even if this means moving the patient to an institution better suited at performing EUS-FNA. If the first attempt was EUS-FNA, reassessment of the EUS findings and other imaging should be undertaken, followed by a review of the FNA line of approach. Although repeat EUS-FNA is a costly procedure, it is still a less expensive and less invasive option than biopsy via laparoscopy or laparotomy. ${ }^{10,14}$

Repeat EUS-FNA of cystic lesions of the pancreas should be considered carefully as it has been recommended that only one draining pass be made because of risks as high as $14 \%$ of infection even if there is no cellular cytological material. Correlation with imaging is mandatory. ${ }^{15}$ This infection risk can be reduced to under $3 \%$ by using intraprocedural intravenous antibiotics, such as fluoroquinolone and then oral antibiotics for 3-5 days. $^{16-19}$

\section{Negative}

A negative cytology sample is defined as a cytological interpretation that is negative for malignancy and any cellular atypia; preferably a diagnosis is made that is specific for a benign non-neoplastic condition. A descriptive negative interpretation implies that the sample is adequately cellular and that no cytological atypia is identified in the evaluated cytology sample. This includes the presence of normal pancreatic tissue in the appropriate clinical setting such a vague fullness on imaging and no distinct mass lesion. A negative cytology interpretation that is 
descriptive without a diagnosis of a specific condition such as chronic pancreatitis or pseudocyst is not synonymous with a benign lesion.

Entities that fall under this category include acute, chronic, and autoimmune pancreatitis, pseudocyst, ectopic, or intrapancreatic splenule and lymphoepithelial cyst.

The multidisciplinary team or individual in charge of the patient's care must perform the "triple test," i.e., an assessment that includes the clinical presentation, radiologic findings, and cytopathology. If any one of the elements of the "triple test" are discrepant it is mandatory to reassess that component, and if found on review to be sound, then the overall diagnosis and other elements have to be reassessed. , $8,9,13,15$ For example, if EUS demonstrates biliary or pancreatic duct dilation, or radiology finds regional lymphadenopathy, it is more likely that there is a malignancy despite a non-diagnostic or negative cytology report. In such cases the interventionalist might be more aggressive in their attempts at obtaining material via FNA or proceed to laparoscopy or laparotomy. $6,15,20-$ 23 Pancreatic EUS-FNA has a very high specificity with very few false positive interpretations; however, false negative interpretations are not uncommon due to sampling and interpretive errors, which impact the sensitivity of the test.

Postprocedural management of specific benign diagnoses include the following:

Acute Pancreatitis is usually managed by an institutional specific protocol, which commonly consists of cessation of oral intake, intravenous fluid hydration, and narcotic analgesia. Patient recovery is largely monitored by the patient's symptoms and the physican's examination. The etiology of the pancreatitis is sought to prevent future episodes. Significant complications of pancreatitis including pseudocyst, hemorrhage, obstruction, and pancreatic necrosis may occur. Pseudocysts commonly occur in a background of pancreatitis and EUS-FNA may have a significant risk of post procedure infection. Life support may be necessary in severe cases of acute pancreatitis, and surgical intervention with resection and debridement is reserved for cases where medical management fails. Post-ERCP pancreatitis may complicate up to $5 \%$ of ERCP procedures. Most cases are mild but rare fatalities are reported. ${ }^{24}$ Bile duct brushings have been associated with potential exacerbation of acute pancreatitis. EUSFNA is an excellent alternative to ERCP/brushings for evaluation of extra-hepatic bile duct and periductal lesions that appear to arise in the intrapancreatic ducts and gallbladder. ${ }^{12,16,25,26}$

Chronic pancreatitis is characterized by chronic abdominal pain due to progressive inflammation, destruction of pancreatic tissue, parenchymal replacement by fibrous tissue, and resultant impairment of pancreatic exo- crine and endocrine function. Therapy is aimed at identification and removal of the cause of the pancreatitis, treating the patient's pain, and managing pancreatic failure (e.g., enzyme digestive replacement for exocrine failure and oral hypoglycemic or exogenous insulin for endocrine failure). ${ }^{25}$

Autoimmune pancreatitis (AIP) is a rare disease. The radiologic pattern of AIP can be distinctive with a diffusely enlarged pancreas, but may also mimic pancreatic cancer. Elevated serum $\operatorname{IgG}_{4}$, the clinical context and response to steroids may help confirm the diagnosis. Steroids are the main treatment. ${ }^{16,27,28}$ There are no known specific complications of EUS-FNA of autoimmune pancreatitis. $^{29}$

Pseudocysts usually occur in a background of chronic pancreatitis or a history of acute pancreatitis related to trauma. Once diagnosed their management is dependent on their size and symptomatology and presence/absence of ductal communication. Treatment may involve endoscopic stenting, internal drainage (endoscopic or operative), or external drainage (percutaneous). ${ }^{30,31}$ EUS-FNA may have a significant risk of postprocedural infection. ${ }^{16,18,24}$

Lymphoepithelial cysts are relatively rare lesions that can be managed conservatively if the patient is asymptomatic and the diagnosis can be securely established by imaging and EUS-FNA. They need to be distinguished from mucinous cystic lesions of the pancreas. EUS-FNA can establish the diagnosis based on the presence of benign squamous cells, keratotic debris, and the lack of mucin. There are no specific complications of FNA of lymphoepithelial cysts. ${ }^{13,32}$

Accessory spleen or splenules (splenucules) can occur in the pancreas or in the splenic hilum adjacent to the pancreas. They are commonly found incidentally on CT radiologic examination and even with EUS may raise a differential diagnosis that includes neoplasms. FNA can be diagnostic. No complications (hemorrhage) have been reported with FNA. If a clinical question remains after identification or the diagnosis is uncertain, resection is curative.

Hydatid cysts have been reported in the pancreas or impinging on the pancreas. FNA poses a potential risk of an anaphylactic reaction following leakage of cyst contents and activation of Type 1 hypersensitivity. FNA where the diagnosis was suspected prior to the procedure based on imaging findings has not been reported, and the actual risk with FNA is not known. Therapy is careful surgical resection. ${ }^{18}$

\section{Atypical}

An atypical interpretation is defined as cytoplasmic, nuclear, or architectural features that are not consistent with normal or reactive cellular components of the pancreas or bile ducts, but features are not sufficient to 
indicate a neoplasm or overt malignancy. This interpretation calls for additional diagnostic testing.

In other organ systems the usual response to an atypical cytologic interpretation is a repeat procedure. For uterine cervical cytology or thyroid FNA the performance of a repeat procedure is relatively easy with the major problem being rescheduling of the patient back into the clinic. The logistics of repeat EUS, ERCP, or percutaneous FNA are much more involved and require the services of a number of individuals and utilization of expensive equipment. The resource utilization and costs of operative biopsy are even greater. The resource problem causes immediate repeat diagnostic procedure to be a nontrivial issue. The appropriate course of action is dependent on a multidisciplinary review, the functional status of the patient and the wishes of the patient after clinical consultation.

Ancillary testing in some cases may assist determination of the management of the patient after an atypical cytological diagnosis. Just as positivity for high-risk human papilloma virus increases the likelihood of disease in an indeterminate "atypical" cervical smear, atypical pancreatic cytology in combination with a number of biochemical tests may be helpful in clinical management and follow-up. Although not routinely performed, Dpc4/ SMAD4 suppressor gene is lost in 55\% of pancreatic ductal adenocarcinomas (PDAC), and this finding may add support to the indeterminate "atypical" interpretation when imaging is suspicious for adenocarcinoma. Although not specific for malignancy, detection of mutant $K R A S$, which is seen in over $90 \%$ of malignancies may contribution to management decisions in the appropriate clinical setting. ${ }^{33-35}$

\section{Neoplastic}

Neoplastic: Benign. The major entity in this category, serous cystic neoplasm (SCN), can be observed or treated by resection. SCN may or may not have a diagnostic imaging presentation. When it does, FNA is not performed. When there is uncertainty about the diagnosis, FNA is performed in an attempt to make a specific diagnosis on the one hand, but to at least make a diagnosis of a nonmucinous cyst. If imaging, cytology, and cyst fluid biochemistry (CEA and amylase) support an interpretation of a SCN, the patient can be conservatively managed with observation, with the proviso that the patient is asymptomatic and that there is no evidence of significant growth, which raises the risk of hemorrhage and rupture. ${ }^{17,18,36,37}$

Neoplastic: Other. Pancreatic neuroendocrine tumor (PanNET). PanNET present two problems: tumor growth and spread and hormonal activity. Whether to perform surgery and the type of surgery is dependent on patient age, fitness, and symptoms as well as lesion loca- tion, size, grade, and stage. With the increasing use of cross-sectional imaging, very small $(\sim 1 \mathrm{~cm})$ tumors are being discovered incidentally in a large number of patients, many of whom are elderly and with comorbid conditions increasing surgical risk. PanNETs may grow very slowly for prolonged periods, and although the majority (50-60\%) eventually exhibits malignant behavior, surgical intervention may not be the best option for all patients. As such, placing PanNETs in this more generic category of Neoplastic: Other rather than in the positive or malignant category increases management options significantly. Convincing a patient that conservative management of their incidental $1 \mathrm{~cm}$ PanNET is the best option for them is virtually impossible when diagnosed by cytology as malignant.

Genetic testing for germline mutations should be performed if the family or personal history is suggestive of Multiple Endocrine Neoplasia (MEN 1) or Von Hippel Lindau Disease. Symptoms usually cause functioning PanNETs to be discovered at a smaller size than with nonfunctioning PanNETs. Controlling the symptoms initially treats symptomatic, functioning PanNET's. For example, proton pump inhibitors or high dose histamine H2-receptor antagonists can oppose hypersecretion of gastrin in Zollinger-Ellison syndrome. Secretion of other hormones may be controlled with somatostatin analogs (Octreotide). Where resection has failed or is impossible, a number of molecularly based modern treatments are available including sunitinib (tyrosine kinase inhibitor), rapmycyin (mTOR inhibitor), and PRRt (peptide receptor radionuclide therapy). ${ }^{6,38}$ Functioning PanNET may have elevated serum pancreatic polypeptide (PP), insulin, Cpeptide, proinsulin, gastrin, vasoactive intestinal peptide (VIP), glucagon, calcitonin, or somatostatin. Levels for these hormones should be drawn and monitored during the therapeutic process. For patients with nonfunctioning PanNET, serum chromogranin A, is useful for following treatment response. Pancreatic polypeptide may also be elevated in apparently nonfunctional PanNET and can serve as a useful post-treatment marker. ${ }^{39}$ Local and or hepatic resection is done for functioning and nonfunctioning tumors with the aim of curative resection or debulking/palliation dependent upon size and location. The National Comprehensive Cancer Network ${ }^{\circledR}\left(\mathrm{NCCN}^{\circledR}\right)$ provides guidelines and algorithms for management of functioning and nonfunctioning PanNETs that are available online. ${ }^{40-44}$

\footnotetext{
"http://www.nccn.org/professionals/physician_gls/f_guidelines.asp\#neuroendocrine. All rights reserved. To view the most recent and complete version of the guidelines, go online to www.nccn.org. NATIONAL COMPREHENSIVE CANCER NETWORK $®, \quad N C C N ®, \quad N C C N$ GUIDELINES $®$, and all other NCCN Content are trademarks owned by the National Comprehensive Cancer Network
} 
For patients with metastatic disease, if the metastatic disease is restricted to the liver, resection (including the possibility of hepatectomy and transplantation) is warranted for both functioning and nonfunctioning tumors with the aim of curative resection or debulking/palliation dependent on the size and location of the tumor. Embolization or radiofrequency ablation may be considered with or without chemotherapy for liver metastases to decrease symptoms from hormonally active tumors. Hepatic transplantation may be considered in select cases. Somatostatin analogs infusion may be indicated in resection of primary tumors, metastatic lesions, anesthetic procedures, or embolization to avoid hormonal crises. The $\mathrm{NCCN}^{\circledR}$ management algorithm for metastatic disease that provides detail is also accessible via the weblink provided below. ${ }^{\dagger 44,45}$

Solid-pseudopapillary neoplasm (SPN). Surgical resection is the treatment for solid-pseudopapillary neoplasm. Approximately $15-20 \%$ are malignant with local recurrence and distant spread most commonly to the liver. Most patients are cured by resection with $95 \%$ or better survival at five years. Even with hepatic and lymph node metastases, the tumor shows low aggressiveness. ${ }^{18,46,47}$

Mucinous cystic neoplasm. Surgical resection is the treatment of choice for all $\mathrm{MCN}$ regardless of grade due to the expense and anxiety associated with lifelong surveillance of the usual middle-aged female affected by this neoplasm. Although most are benign, nearly $18 \%$ will undergo malignant transformation. Mural nodularity and cyst diameter greater than $4 \mathrm{~cm}$ are imaging features associated with malignant risk. Patients considered surgical risks with small cysts without high-risk imaging features may be managed with observation, but must be subjected to lifelong clinical and radiologic scrutiny to evaluate for features suggestive of malignant transformation. ${ }^{46,48,49}$

Intraductal papillary mucinous neoplasms (IPMN). Surgical intervention versus observation depends on the type of IPMN: IPMN that involve the main duct alone or in combination with branch-duct cysts (M-IPMN) or branch duct (BD-IPMN) only cysts. ${ }^{49-51}$

M-IPMNs are treated by resection. Observation of mucous extruding from a patulous ("fish-mouth") Ampulla of Vater is diagnostic of M-IPMN. Main-duct dilatation $>10 \mathrm{~mm}$ is considered a high-risk imaging feature and surgery is typically performed without preoperative tissue diagnosis. M-IPMN's are most commonly lined by intestinal-type epithelial cells and invasive carcinoma is found in 40-45\% with high-grade dysplasia identified in another $20 \%{ }^{52}$ M-IPMN's usually occur in the pancreatic head requiring a pancreatoduodenectomy.

Management options for patients with BD-IPMN are more controversial. BD-IPMNs are most often benign and asymptomatic. Increased use of cross-sectional imaging has greatly increased the detection of these incidentalomas identified in nearly $8 \%$ of elderly patients. Since, the time to malignant transformation is estimated to be about 10 years, many patients, especially those with comorbid conditions, benefit more by conservative observation than surgery. The decision to operate is determined by the risk of malignancy, which in many centers is solely based on imaging features such as the presence of an enhancing mural nodule. Worrisome imaging features such as large cyst size $(>3 \mathrm{~cm})$ or nonenhancing mural nodule warrant evaluation with FNA. ${ }^{49}$ The cytological evaluation of the cyst contents for high-grade epithelial atypia (e.g., highgrade dysplasia or invasive carcinoma) is a high-risk feature as well warranting surgical resection. ${ }^{53}$ Although the typical BD-IPMN is lined by low-grade dysplastic gastric type epithelium, when invasive carcinoma occurs, it is of the tubular type with the same dismal prognosis as conventional ductal adenocarcinoma. ${ }^{54}$ As such, the patient has the best prognosis when premalignant (e.g., preinvasive) carcinoma is identified, preferably at the level of high-grade dysplasia. Demographics (age/gender), family history of pancreatic cancer, social history of tobacco usage, obesity, and serum tumor markers (CA19-9, Ha1c) may also influence the patient's risk of pancreatic cancer and, thus, the decision to operate.

A flowchart illustrating the Fukuoka revised guidelines for management of mucinous cystic neoplasms and intraductal papillary mucinous neoplasms is shown with permission in Figure $1 .^{49}$

Gastrointestinal stromal tumor (GIST). For localized GIST, surgical resection with an aim of achieving negative margins is indicated. For advanced or metastatic c$\mathrm{Kit}+\mathrm{GIST}$, the c-Kit tyrosine kinase inhibitor (TKI), Imatinib, has shown improved clinical response over what was previously a dismal outlook. Unfortunately, TKI therapy is not a cure and resistance tends to develop. Thus few patients with advanced disease are cured by chemotherapy and remission or stabilization is the current expectation until new generations of therapeutic agents are created and tested.

\section{Suspicious}

These lesions show severe cellular atypia, suspicious for invasive ductal carcinoma or other high-grade malignant neoplasm, for example, an aspirate with a solid-cellular smear pattern without diagnostic cytological features or tissue available for confirmatory molecular or immunohistochemical findings supportive of a specific neoplasm such as PanNET or SPN, which would then allow for the classification as "Neoplastic: Other."

As with the benign and atypical interpretations, when there are discrepant imaging findings suggesting malignancy, and an atypical interpretation, management of the patient with a "suspicious" cytological interpretation requires a stringent multidisciplinary review of the clinical and imaging findings. Biochemical and molecular 


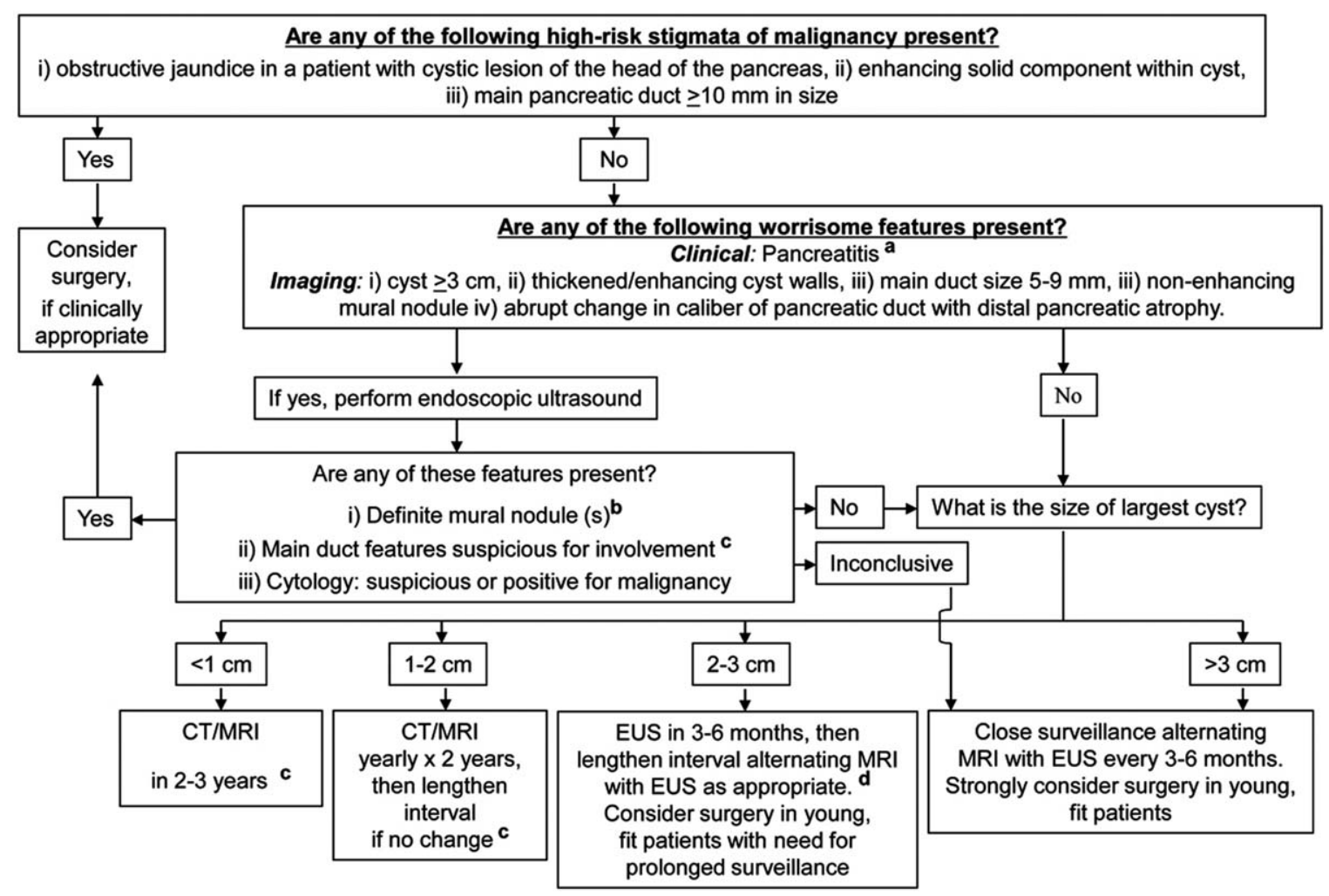

Reprinted from Pancreatology, Vol. 12, 2012, Tanaka, M., et al, International Consensus Guidelines 2012 for the management of IPMN and MCN of the Pancreas. Pages 183-197., Copyright (2012), with permission from Elsevier

Fig. 1. The Fukuoka revised guidelines for management of mucinous cystic neoplasms and intraductal papillary mucinous neoplasms. ${ }^{49}$

analytical markers may increase the sensitivity and specificity of the interpretation.

If the cytological findings are unexpected and do not correlate with imaging predictions of a benign lesion, then repeat brushings or EUS-FNA should be considered, with the goal of obtaining sufficient tissue for cellblock preparation and ancillary testing.

If the cytological findings of suspicious for malignancy do correlate with the imaging findings suggestive of malignancy, then the patient can be worked up and staged. This could include laparoscopy with biopsy, further EUS-FNA of lymph nodes, PET, and CT scans to exclude distant metastases. The exact workup is dependent on the availability of these modalities, personnel, and local established protocols.

\section{Positive/Malignant}

The malignant category is definitive for a high-grade malignancy and should always be accompanied by a specific diagnosis whenever possible. ${ }^{55}$

Management relates to the specific type of malignancy present. Since, conventional ductal adenocarcinoma
(PDAC) or a variant represents 9 of 10 pancreatic malignancies, this interpretation category, more likely than not, represents PDAC. In some cases, it may not be possible to define the particular malignancy on cytology alone. In these cases ancillary tests on cell block or other fluid based preparations should be done, when available. For example, it is important to distinguish acinar cell carcinoma, a high-grade aggressive malignancy, from mimickers such as PanNET. Metastatic malignancy to the pancreas should always be considered especially when the imaging or EUS findings are not typical of a pancreatic primary or the cytological findings are not characteristic.

Adenocarcinoma of the Pancreatobiliary Ducts, and Variants

The current NCCN Clinical Practice Guidelines In Oncology (NCCN Guidelines ${ }^{\circledR}$ ) for the management of patients with PDAC are outlined in Tempero et al. ${ }^{56}$ Currently, $\sim 15 \%$ of patients are candidates for surgical resection based upon preoperative staging. Using all available diagnostic modalities including high resolution, dual phase 
CT scanning, and EUS, the accuracy of determining resectability is $\sim 85 \%$, but this still leaves one in five laparotomies that will find a nonresectable tumor. Initial laparoscopy may be of further help for detection of occult metastases reducing the number of unnecessary laparotomies to $10 \%$ for body/tail lesions, which tend to be more advanced at presentation than head/uncinate lesions.

Prior to attempted resection, liver "function tests" including coagulation tests are necessary to determine the patient's functional liver reserve. If liver function tests fail to return to normal following endoscopic stenting in patients with biliary obstruction, this may indicate liver failure from prolonged biliary obstruction or unsuspected pre-existing liver disease.

Tumors in the head or uncinate process of the pancreas are treated with pylorus preserving pancreaticoduodenectomy (Whipple's procedure). Tumors of the body/tail are treated with distal pancreatectomy and splenectomy. With surgical resection and adjuvant chemotherapy patients have a median survival of 18-24 months and a 15-20\% chance of 5-year survival in this limited form of disease.

Serum CA19-9 is elevated in $70-80 \%$ of cases of pancreatic ductal adenocarcinoma. While not an optimal screening tool, it is useful to monitor for recurrence and disease progression in patients demonstrating preoperative elevated serum CA19-9.

In cases of nonresectable disease, common complications include pain, jaundice, pancreatic exocrine insufficiency, and gastric outlet obstruction. Oral narcotic analgesics and celiac nerve blocks may address the pain when it is due to invasion of the pancreatic nerves and celiac plexus.

Endoscopic stents are optimal palliation for biliary obstruction. Stents obstructed by tumor ingrowth can be restented. Operative biliary bypass may be considered in cases where patients are explored for cure and found to be locally unresectable.

Pancreatic exocrine insufficiency may be managed with exogenous pancreatic enzyme supplementation. Gastric outlet obstruction is optimally managed with newer generation endoscopic duodenal stents. If not amenable to endoscopic stenting, operative gastrojejunal bypass may be considered in fit patients and a gastrostomy tube may be placed for palliation in unfit patients.

Less than $10 \%$ of tumors resected are Stage I or II. Overall 5 year survival for all patients with pancreatic adenocarcinoma is $\sim 5 \%$ (SEER data 1996-2003). ${ }^{49}$ Chemotherapeutic agents gemcitabine, 5-flourouracil, and paclitaxel have each been shown to improve survival alone or in combination. Adjuvant gemcitabine on average increases patient survival 4-6 weeks. Gemcitabine treatment for unresectable disease also improves survival several weeks. A recent study indicates this effect may be enhanced by combination treatment with paclitaxel. Neoadjuvant trials have shown slight benefit. ${ }^{57}$
Approximately $10 \%$ of patients who develop pancreatic cancer have a hereditary component. Another $10 \%$ of patients who develop pancreatic cancer may be predisposed by a precancerous (mucinous) pancreatic cystic lesion. Patients with mucinous pancreatic cysts or a familial predisposition to pancreatic cancer, may benefit from screening. Early detection may prevent pancreatic cancer or detect its presence early enough to optimize the chance of curative treatment. At present, prevention in these select groups is the most promising treatment.

\section{Acinar Cell Carcinoma}

These tumors are managed surgically and medically in a similar fashion to pancreatic ductal adenocarcinoma.

\section{High-Grade Neuroendocrine Carcinoma (Small and Large Cell Type)}

These tumors are associated with a rapid clinical course with the majority of patients having liver metastases at the time of diagnosis. Treatment is with chemotherapy regimens similar to small cell lung carcinoma guidelines. $^{58,59}$

\section{Pancreatoblastoma}

The optimum management of this malignant tumor of childhood is complete surgical resection. Approximately half do well with surgical resection. In cases of resectable disease, adjuvant chemotherapy is recommended with regimens including cisplatin and doxorubicin. Even following what appears to be curative resection, there is a recurrence rate and patients must be followed closely. In cases of unresectable tumor at initial diagnosis, neoadjuvant chemotherapy may downstage the tumor enough to reconsider surgery. ${ }^{60}$

\section{Lymphoma}

Lymphomas are included in this category although not all lymphomas are "high-grade" per se. Specific treatment will be subject to medical management directed by the hematologic workup including histologyic morphology, flow cytometry, and cytogenetic analysis.

\section{Metastases}

The presence of metastatic disease usually indicates widespread dissemination of the primary malignancy and the management will be dependent on the specific primary tumor and its histologic grade.

\section{Summary}

These guidelines provide a brief overview for patient treatment and management following the cytological interpretation of diseases as categorized by the six-tiered terminology and nomenclature classification system proposed by Committee III (refer to document from 
committee III in this issue). More detailed treatment strategies are provided by surgical and gastrointestinal specialty organizations [British society of Gastroenterology, Pancreatic Society of Great Britain, Royal College of Pathology, Japan Pancreas Society, International Association of Pancreatology] $]^{60,61}$ as well as NCCN.

\section{References}

1. Elek G, Gyokeres T, Schafer E. Early diagnosis of pancreatobiliary duct malignancies by brush cytology and biopsy. Pathol Oncol Res 2005;11:145-155.

2. Eloubeidi MA, Jhala D, Chhieng DC, et al. Yield of endoscopic ultrasound-guided fine-needle aspiration biopsy in patients with suspected pancreatic carcinoma. Cancer Cytopathol 2003;99:285-292.

3. Stewart CJ, Mills PR, Carter R, et al. Brush cytology in the assessment of pancreatico-biliary strictures: A review of 406 cases. J Clin Pathol 2001;54:449-455.

4. Kocjan G, Rode J, Lees WR. Percutaneous fine needle aspiration of cytology of the pancreas. J Clin Pathol 1989;42:341-347.

5. Chong I. Cunningham. Pancreatic cancer. In: Longo, editor. Harrison's principles of internal medicine. 19 ed. New York, NY: McGraw Hill; p 786-789.

6. Spier B, Johnson E, Gopal D, et al. Predictors of malignancy and recommendedfollow-up for patients with negative endoscopicultrasound-guided fine-needle aspiration of suspectedpancreatic lesions. Can J Gastroenterol 2009;23:279-286.

7. Wani S, Gupta N, Gaddam S, et al. A comparative study of endoscopic ultrasound guided fine needle aspiration with and without a stylet. Dig Dis Sci 2011;56:2409-2414.

8. Gimeno-García AZ, Elwassief A. How to improve the success of endoscopic ultrasound guided fine needle aspiration cytology in the diagnosis of pancreatic lesions. J Interv Gastroenterol 2012;2:3136.

9. Noh KW, Wallace MB. Endoscopic ultrasound-guided fine-needle aspiration in the diagnosis and staging of pancreatic adenocarcinoma. Medscape General Med 2005;7:1-5.

10. Suzuki R, Lee JH, Krishna SG, et al. Repeat endoscopic ultrasound-guided fine needle aspiration for solid pancreatic lesions at a tertiary referral center will alter the initial inconclusive result. J Gastrointest Liver Dis 2013;22:183-187.

11. Gagovic V, Spier B, DeLee R, et al. Endoscopic ultrasound fineneedle aspiration characteristics of primary adenocarcinoma versus other malignant neoplasms of the pancreas. Can J Gastroenterol 2012;26:691-696.

12. Xie HB, Cornwell R, Grossman JE, Hoerl HD, Kurtycz DFI. Bronchoscopy-guided transtracheal and transbronchial fine-needle aspiration biopsy: A 5-year institutional review of 111 cases. Diagn Cytopathol [Internet]. 2002;27:276-281. Available from: http://doi. wiley.com/10.1002/dc.10187.

13. Karim Z, Walker B, Lam E. Lymphoepithelial cysts of the pancreas: The use of endoscopic ultrasound-guided fine-needle aspiration in diagnosis. Can J Gastroenterol 2010;24:348-350. Available from: http://pubget.com/paper/20559575? institution $=$.

14. Rosch T, Hofrichter K, Frimberger E, et al. ERCP or EUS for tissue diagnosis of biliary strictures? A prospective comparative study. Gastrointest Endosc 2004;60:390-396.

15. Wiersema MJ, Vilmann P. EUS-guided FNAB. Gastrointest Endosc 1997;45:243-250.

16. Reddy N, Wilcox CM, Tamhane A, Eloubeidi MA, Varadarajulu S. Protocol-based medical management of post-ERCP pancreatitis. J Gastroenterol Hepatol 2008;23:385-392.

17. Del Chiaro M, Verbeke C, Salvia R, et al. European experts consensus statement on cystic tumours of the pancreas. Dig Liver Dis 2013.
18. Farrell JJ, Fernández-del Castillo C. Pancreatic cystic neoplasms: Management and unanswered questions. Gastroenterology 2013; 144:1303-1315.

19. Lee LS, Saltzman JR. EUS-guided FNA of pancreatic cysts. Clin Gastroenterol Hepatol 2005;3:231-236.

20. Eloubeidi MA, Tamhane A. EUS-guided FNA of solid pancreatic masses: A learning curve with 300 consecutive procedures. Gastrointest Endosc 2005;61:700-708.

21. Eloubeidie MA, Chen VK. EUS-guided FNA of suspected cholangiocarcinoma [Internet]. 2004 ed. Clin Gastroenterol Hepatol 2004; 2:209-213. Available from: http://sfx.wisconsin.edu/wisc?sid= Entrez:PubMed\&id=pmid: 15017604 .

22. Klapman JB, Logrono R, Dye CE, Waxman I. Clinical impact of on-site cytopathology interpretation on endoscopic ultrasoundguided fine needle aspiration. Am J Gastroenterol 2003;98:12891294.

23. Moparty B, Logrono R, Nealon WH, Waxman I, Raju GS, Pasricha PJ. The role of endoscopic ultrasound and endoscopic ultrasoundguided fine-needle aspiration in distinguishing pancreatic cystic lesions. Diagn Cytopathol 2007;35:18-25.

24. Eloubeidi MA, Tamhane A, Varadarajulu S, Wilcox CM. Frequency of major complications after EUS-guided FNA of solid pancreatic masses: A prospective evaluation. Gastrointest Endosc 2006; 63:622-629.

25. Trikudanathan G, Navaneethan U, Vege SS. Modern treatment of patients with chronic pancreatitis. Gastroenterol Clin North America 2012;41:63-76.

26. Erickson RA, Garza AA. EUS with EUS-guided fine-needle aspiration as the first endoscopic test for the evaluation of obstructive jaundice. Gastrointest Endosc 2001;53:475-484.

27. Sugumar A. Diagnosis and management of autoimmune pancreatitis. Gastroenterol Clin North Am 2012;41:9-22. Available from: http://dx.doi.org/10.1016/j.gtc.2011.12.008.

28. Erickson RA, Garza AA. EUS and EUS-guided FNA as first endoscopic test for the evaluation of obstructive jaundice. Gastrointest Endosc 2001;53:475-484.

29. Sugumar A. Diagnosis and management of auto. Gastroenterol Clin North America 2012;41:9-22.

30. Trikudanathan G, Navaneethan U, Vege SS. Modern treatment of patients with chronic pancreatitis. Gastroenterol Clin North Am 2012;41:63-76. Available from: http://dx.doi.org/10.1016/j.gtc.2011. 12.009 .

31. Basturk O, Coban I, Adsay NV. Pancreatic cysts: Pathologic classification, differential diagnosis, and clinical implications. Arch Pathol Lab Med 2009;133:423-438. Available from: http://eutils.ncbi. nlm.nih.gov/entrez/eutils/elink.fcgi?dbfrom $=$ pubmed\&id $=19260748 \&$ retmode $=$ ref $\& \mathrm{cmd}=$ prlinks.

32. Nasr J, Sanders M, Fasanella K, Khalid A, McGrath K. Lymphoepithelial cysts of the pancreas: an EUS case series. Gastrointest Endosc 2008;68:170-173.

33. Maitra A, Kern SE, Hruban RH. Molecular pathogenesis of pancreatic cancer. Best Pract Res Clin Gastroenterol 2006;20:211-226.

34. Hruban RH, Adsay NV. Molecular classification of neoplasms of the pancreas. Hum Pathol 2009;40:612-623. Available from: http:// dx.doi.org/10.1016/j.humpath.2009.01.008.

35. Wilentz RE, Iacobuzio-Donahue CA, Argani P, et al. Loss of expression of Dpc4 in pancreatic intraepithelial neoplasia: Evidence that DPC4 inactivation occurs late in neoplastic progression. Cancer Res 2000;60:2002-2006.

36. Tseng JF, Warshaw AL, Sahani DV, Lauwers GY, Rattner DW, Castillo CF-D. Serous cystadenoma of the pancreas. Ann Surg 2005; 123:111-118.

37. Brandwein SL, Farrell JJ, Centeno BA, Brugge WR. Detection and tumor staging of malignancy in cystic, intraductal, and solid tumors of the pancreas by EUS. Gastrointest Endosc 2001;53:722-727. 
38. Ito T, Igarashi H, Jensen RT. Therapy of metastatic pancreatic neuroendocrine tumors (pNETs): Recent insights and advances. J Gastroenterol [Internet]. 2012;47:941-960. Available from: http://eutils.ncbi. nlm.nih.gov/entrez/eutils/elink.fcgi?dbfrom = pubmed\&id $=22886480$ $\&$ retmode $=$ ref $\& \mathrm{cmd}=$ prlinks.

39. Syversen U, Ramstad H, Gamme K, Qvigstad G, Falkmer S, Waldum HL. Clinical significance of elevated serum chromogranin A levels. Scand J Gastroenterol [Internet]. 2004;39:969-973. Available from: http://informahealthcare.com/doi/abs/10.1080/00365520410003362.

40. Klimstra DS. Pathology reporting of neuroendocrine tumors: Essential elements for accurate diagnosis, classification, and staging. Semin Oncol 2013;40:23-36.

41. Klimstra DS, Modlin IR, Coppola D, Lloyd R, Suster S. The pathologic classification or neuroendocrine tumors [Internet]. Pancreas 2010; 39:707-712. Available from: http://sfx.wisconsin.edu/wisc ?sid=Entrez:PubMed\&id=pmid:20664470.

42. Couvelard A, Deschamps L, Ravaud P, et al. Heterogeneity of tumor prognostic markers: A reproducibility study applied to liver metastases of pancreatic endocrine tumors. Mod Pathol 2008;22: 273-281.

43. Yang Z, Tang LH, Klimstra DS. Effect of tumor heterogeneity on the assessment of Ki67 labeling index in well-differentiated neuroendocrine tumors metastatic to the liver: Implications for prognostic stratification. Am J Surg Pathol 2011;35:853-860.

44. NCCN Clinical Practice Guidelines in Oncology (NCCN Guidelines $($ ) for Neuroendocrine Tumors V.2.2014. (C) National Comprehensive Cancer Network, Inc 2013. Available at: http://www.nccn. org/professionals/physician_gls/f_guidelines.asp\#neuroendocrine. Accessed December 20, 2013.

45. Plockinger U, Rindi G, Arnold R, et al. Guidelines for the diagnosis and treatment of neuroendocrine gastrointestinal tumours. Neuroendocrinology 2004;80:394-424.

46. Fisher W, Andersen D, Bell R, Saluja A. Pancreas. In: Brunicardi C, editor. Schwartz's principles of surgery. 9 ed. New York: McGraw-Hill; 2010. p 1217-1237.

47. Abraham SC. DSKREWCJYKCMBJLCT-TWRHH. Solidpseudopapillary tumors of the pancreas are genetically distinct from pancreatic ductal adenocarcinomas and almost always harbor $\beta$ catenin mutations. Multiple values selected. Am Soc Invest Pathol 2002;160:755-765.

48. Basturk O, Coban I, Adsay NV. Pancreatic cysts: Pathologic classification, differential diagnosis, and clinical implications. Arch Pathol Lab Med 2009;133:423-438. sfx.wisconsin.edu.

49. Tanaka M, et al. International consensus guidelines 2012 for the management of IPMN and MCN of the pancreas. Pancreatology 2012;12:183-197.

50. Pitman MB. Revised international consensus guidelines for the management of patients with mucinous cysts. Cancer Cytopathology 2012;120:361-365.
51. Pitman MB, Lewandrowski K, Shen J, Sahani D, Brugge W, Fernández-del Castillo C. Pancreatic cysts. Cancer Cytopathol 2009; 118:1-13.

52. Fisher W, Andersen D, Bell R, Saluja A. Pancreas. In: Brunicardi C, editor. Schwartz's principles of surgery. 9 ed. New York: McGraw-Hill; 2010. p 1217-1237.

53. Pitman MB, et al. High-grade atypical epithelial cells in pancreatic mucinous cysts are a more accurate predictor of malignancy than "positive" cytology. Cancer Cytopathol 2010;118:434-440.

54. Mino-Kenudson M, et al. Prognosis of invasive intraductal papillary mucinous neoplasm depends on histological and precursor epithelial subtypes. Gut 2011;60:1712-1720.

55. Eltoum IA, Eloubeidi MA, Chhieng DC, et al. Cytologic grade independently predicts survival of patients with pancreatic adenocarcinoma. Am J Clin Pathol 2005;124:697-707.

56. Tempero MA, Arnoletti JP, Behrman SW, et al. Pancreatic adenocarcinoma guidelines, Version 2.2012. J Natl Compr Canc Netw 2012;10:703-713.

57. Gillen S, Schuster T, Meyer zum Bü̈chenfelde C, Friess H, Kleef J. Preoperative/neoadjuvant therapy in pancreatic cancer: A systematic review and meta-analysis of response and resection percentages. Res Dev Disabil 2010;7:e1000267.

58. Yachida S, Vakiani E, White CM, et al. Small cell and large cell neuroendocrine carcinomas of the pancreas are genetically similar and distinct from well-differentiated pancreatic neuroendocrine tumors. Am J Surg Pathol 2012;36:173-184.

59. Roland CL, Bian A, Mansour JC, et al. Survival impact of malignant pancreatic neuroendocrine and islet cell neoplasm phenotypes. J Surg Oncol 2012;105:595-600.

60. Yamaguchi K, Tanaka M, Committee for Revision of Clinical Guidelines for Pancreatic Cancer of Japan Pancreas Society. EBM based clinical guidelines for pancreatic cancer 2009 from the Japan Pancreas Society: A synopsis. Jpn J Clin Oncol 2011;41:836840.

61. Pancreatic Section of the British Society of Gastroenterology, Pancreatic Society of Great Britain and Ireland, Association of Upper Gastrointestinal Surgeons of Great Britain and Ireland, Royal College of Pathologists, Special Interest Group for Gastro-Intestinal Radiology. Guidelines for the management of patients with pancreatic cancer periampullary and ampullary carcinomas. Gut 2005; 54(Suppl V):v1-v16. doi: 10.1136/gut.2004.057059.

62. Tanaka M, Castillo CF-D, Adsay V, et al. et al. International consensus guidelines 2012 for the management of IPMN and MCN of the pancreas. Pancreatology 2012;12:183-197.

63. Genevay M, Mino-Kenudson M, Yaeger K, et al. Cytology adds value to imaging studies for risk assessment of malignancy in pancreatic mucinous cysts. Ann Surg 2011;254:977-983.

64. Hruban RH, Adsay NV. Molecular classification of neoplasms of the pancreas. Hum Pathol 2009;40:612-623. 